\title{
Letter to the Editor on the original article: Lower prevalence of congenital cytomegalovirus infection in Portugal: possible impact of COVID-19 lockdown?
}

\author{
María Ríos-Barnés ${ }^{1}$ Melissa Andrea Fontalvo ${ }^{2} \cdot$ Nerea Liñán ${ }^{3} \cdot$ Montserrat Plana $^{2} \cdot$ María Moreno $^{2}$. \\ Cristina Esteva $^{3,4}$. Carmen Muñoz-Almagro ${ }^{3,4,5} \cdot$ Antoni Noguera-Julian ${ }^{1,4,6,7}(\mathbb{D}) \cdot$ Ana Alarcón ${ }^{2,7} \cdot$ CRIB Study team
}

Received: 11 December 2021 / Revised: 11 December 2021 / Accepted: 16 December 2021 / Published online: 29 January 2022

( ) The Author(s), under exclusive licence to Springer-Verlag GmbH Germany, part of Springer Nature 2021

Keywords Behavioral interventions · Congenital cytomegalovirus infection · COVID-19 · Lockdown

\section{Abbreviations \\ cCMV Congenital Cytomegalovirus infection}

\section{Dear Editor,}

We read with interest the article by Fernandez et al. [1], in which the authors describe an eightfold decrease (from 0.67 to $0.078 \%$ ) in the prevalence of congenital Cytomegalovirus infection (cCMV) in Portugal during the COVID-19 pandemic. We would like to share the preliminary results of an ongoing universal screening study of cCMV in Hospital Sant Joan de Déu (Barcelona, Spain). The study was approved by the local Ethics Committee (ref. PIC-205-19); informed consent from parents is obtained at inclusion. cCMV screening is performed in saliva samples, obtained using flocked swabs (catalog no. 300267, Deltalab; Rubí, Spain) in the first 14 days of life, by means of the Alethia CMV DNA amplification assay (Meridian Bioscience; Cincinnati, $\mathrm{OH}$ ) [2]; confirmation of cCMV is performed

Communicated by Peter de Winter

Antoni Noguera-Julian and Ana Alarcón contributed equally to this work.

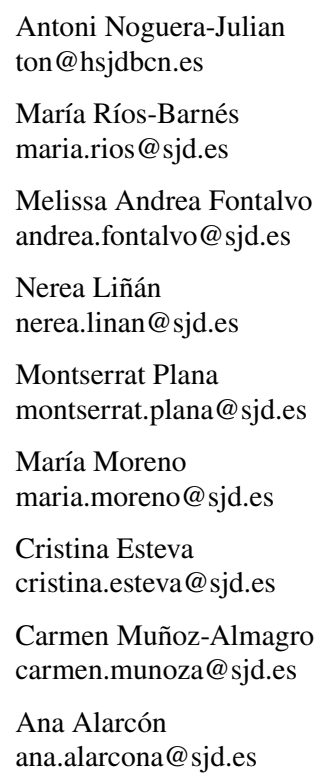

1 Infectious Diseases Unit, Department of Pediatrics, Infectious Diseases and Systemic Inflammatory Response in Pediatrics, Institut de Recerca Sant Joan de Déu, Hospital Sant Joan de Déu, Barcelona, Spain

2 Department of Neonatology, Institut de Recerca Sant Joan de Déu, Hospital Sant Joan de Déu, Barcelona, Spain

3 Department of Molecular Microbiology, Institut de Recerca Sant Joan de Déu, Hospital Sant Joan de Déu, Barcelona, Spain

4 Center for Biomedical Network Research On Epidemiology and Public Health (CIBERESP), Madrid, Spain

5 Department of Medicine, Universitat Internacional de Catalunya, Barcelona, Spain

6 Translational Research Network in Pediatric Infectious Diseases (RITIP), Madrid, Spain

7 Department of Pediatrics, University of Barcelona, Barcelona, Spain 
by PCR in urine samples. Overall, 2735 neonates were included during the first 10 months of the study (February 1 to November 30, 2021), 10 tested positive, and only one case was confirmed in urine. Symptomatic disease at birth led to the diagnosis of one further case (out of 63 patients with clinical indication of cCMV investigation). The prevalence of cCMV was $0.071 \%$ (95\% confidence interval: $0.020-0.260 \%)$.

The prevalence of cCMV in our study is very similar to that reported in Portugal, and far from the $0.6 \%$ that has been described in a meta-analysis in high-income countries [3]. Unfortunately, historical estimates of cCMV to compare with from our region are not available, but a recent study in Madrid reported a $0.47 \%$ prevalence rate [4]. It is likely that the lockdown measures and behavioral interventions to reduce SARS-CoV-2 transmission in Spain during 2020-2021 also impacted on CMV transmission and cCMV prevalence. In fact, a dramatic decrease in respiratory syncytial virus infections in the 2020 winter season [5] and a re-emergence of enterovirus D68 after easing the lockdown in 2021 [6] have also been reported, both viruses sharing the contact transmission route with CMV. We agree with Fernandez et al. [1] that our findings reinforce the role of behavioral risk reduction interventions to prevent $\mathrm{cCMV}$ in pregnant women.

\begin{abstract}
Acknowledgements Other members of the CRIB Study team are as follows: Sara Cardelús (Otorhinolaryngology Dept., Hospital Sant Joan de Déu; Barcelona, Spain); Nuria Carreras, María José Coll, Itzel Muste, María Dolores Perán, and María José Troyano (Neonatology Department, Hospital Sant Joan de Déu; Barcelona, Spain); Jesús Díaz (Ophthalmology Department, Hospital Sant Joan de Déu; Barcelona, Spain); Raquel Ferré, Patrícia Pages (Obstetrics Department, Hospital Sant Joan de Déu; Barcelona, Spain); Clàudia Fortuny (Infectious Diseases Unit, Department of Pediatrics, Institut de Recerca Sant Joan de Déu; Barcelona, Spain); and Mónica Rebollo (Radiology Department, Hospital Sant Joan de Déu; Barcelona, Spain).
\end{abstract}

Authors' contributions AA, AN-J, and CM-A contributed to the study conception and design. MR-B, MAF, MP, MM, and AA participated in the inclusion of patients. NL, CE, and CM-A performed and validated microbiological analyses and results. AA was responsible for data collection and analysis. The first draft of the manuscript was written by MR-B, AN-J, and AA, and all authors commented on previous versions of the manuscript. All authors read and approved the final manuscript.

Funding This work was partially funded by research grants from the Carlos III Institute of Health, Ministry of Economy and Competitiveness (Spain), ref. PI17/00359 and PI19/00095. Deltalab supported the cost of the flocked swabs used in this study, but was not involved in the study design; data collection, and analysis, or the writing and submission of the study results for publication. María Ríos-Barnés was supported by a PFIS fellowship grant from the Instituto de Salud
Carlos III (Ministerio of Science, Innovation and Universities; ref. FI20/00237). Antoni Noguera-Julian was supported by "Subvencions per a la Intensificació de Facultatius Especialistes" (Departament de Salut de la Generalitat de Catalunya, Programa PERIS 2016-2020; ref. SLT008/18/00193).

Availability of data and material The data underlying this article will be shared on reasonable request to the corresponding author.

\section{Declarations}

Ethics approval Ethics approval was obtained from Hospital Sant Joan de Déu (Barcelona, Spain) Ethics Committee (reference PIC-205-19).

Consent to participate and for publication Informed consent was obtained from the parents.

Conflict of interest The authors declare no competing interests.

\section{References}

1. Fernandez C, Chasqueira MJ, Marques A, Rodrigues L, Marçal M, Tuna M, Braz MC, Neto AS, Mendes C, Lito D, Rocha P, Vasconcellos G, Menezes MF, Sousa MJ, Nunes C, Paixão P (2021) Lower prevalence of congenital cytomegalovirus infection in Portugal: possible impact of COVID-19 lockdown?. Eur J Pediatr [published online ahead of print]. https://doi.org/10.1007/ s00431-021-04271-0

2. Gantt S, Goldfarb DM, Park A, Rawlinson W, Boppana SB, Lazzarotto T, Mertz LM (2020) Performance of the Alethia CMV assay for detection of cytomegalovirus by use of neonatal saliva swabs. J Clin Microbiol 58:e01951-e02019. https://doi.org/10.1128/JCM.01951-19

3. Kenneson A, Cannon MJ (2007) Review and meta-analysis of the epidemiology of congenital cytomegalovirus (CMV) infection. Rev Med Virol 17:253-276. https://doi.org/10.1002/rmv.53

4. Blázquez-Gamero D, Soriano-Ramos M, Vicente M, Pallás-Alonso CR, Pérez-Rivilla A, García-Álvarez M, Pinilla Martín MT, Freire X, De Vergas J, De Aragón AM, Zamora B, Epalza C, Moraleda C, Rojo P, Prieto L, Fernández-Cooke E, Ruíz-Contreras J, Delgado R, Folgueira MD; for PICCSA Study Group (2020) Prevalence and Clinical Manifestations of Congenital Cytomegalovirus Infection in a Screening Program in Madrid (PICCSA Study). Pediatr Infect Dis J 39:1050-1056. https://doi.org/10.1097/INF.0000000000002808

5. Torres-Fernandez D, Casellas A, Mellado MJ, Calvo C, Bassat Q (2021) Acute bronchiolitis and respiratory syncytial virus seasonal transmission during the COVID-19 pandemic in Spain: a national perspective from the pediatric Spanish Society (AEP). J Clin Virol 145:105027. https://doi.org/10.1016/j.jcv.2021.105027

6. Benschop KS, Albert J, Anton A et al (2021) Re-emergence of enterovirus D68 in Europe after easing the COVID-19 lockdown, September 2021. Euro Surveill 26. https://doi.org/10.2807/15607917.ES.2021.26.45.2100998

Publisher's Note Springer Nature remains neutral with regard to jurisdictional claims in published maps and institutional affiliations. 